\title{
Rhabdomyolysis following an intensive indoor cycling exercise: A series of 5 cases
}

\section{Dear Editor,}

Spin cycling (spinning) is an increasingly popular indoor sport both in Singapore and worldwide. We find a growing trend in cases of spin-induced rhabdomyolysis (SIR) among beginners of this sport presenting to our Emergency Department (ED), all requiring hospitalisation for further treatment.

Spinning is an indoor cycling sport using a specialised stationary bicycle. It is often touted as an optimal aerobic sport, and a quick solution to increase one's metabolic rate and relieve stress. It is sometimes done in a group, with each session lasting 30-60 minutes. The intensity of cycling can be adjusted through cadence (i.e. the rate at which the cyclist pedals) and pedalling resistance.

Exertional rhabdomyolysis is the breakdown of muscle that occurs after excessive or unaccustomed exercise. It is characterised by muscle pain, markedly elevated levels of serum muscle enzyme (e.g. creatine kinase) and myoglobinuria.

We present a case series of 5 patients, all new to the sport, who developed exertional rhabdomyolysis after their spin session in March 2020. They presented to the ED of the Changi General Hospital, Singapore. This study was approved by the SingHealth Institutional Review Board (CIRB 2020/2475).

Cases. The 5 patients' presenting history, clinical features, diagnostic workup, and medical management are as described. All patients were young adults in their 20 s, non-smokers, with no significant comorbidities. Three of them did not exercise regularly (once per month), while 2 did aerobic exercises 3-4 times a week. Four were attending their first spin class, while 1 was attending her second class. In all the cases, the spin sessions were done as a group and in the presence of a class instructor. The spin duration for these patients was 45 minutes.

The patients presented 2-4 days after their spin class. All had a common presenting complaint of bilateral thigh pain. Four also passed brown or dark urine, with the onset occurring 2-3 days after spinning. None of the patients had any intercurrent illnesses, or had any trauma.

All 5 patients were given intravenous fluids and hospitalised. Peak serum creatine kinase (CK) levels for 1 patient remained at $>100,000 \mathrm{U} / \mathrm{L}$ for 3 days, while peak levels for the rest ranged 11,000-65,000U/L. None presented with, nor developed renal impairment or compartment syndrome. The average length of stay was 2.4 days (range 1-4 days). All patients had decreasing serum CK levels, made good recovery and were well on discharge. Their demographic and clinical data are presented in Table 1.

Discussion. Spinning is a high-intensity workout, with a typical session lasting 30-60 minutes. A vigorous 30 -minute class can burn as much as $300-500$ calories. SIR has been documented after just 15 minutes into a spinning session in an untrained person. ${ }^{1}$

A spin bicycle, compared to a stationary exercise bicycle, closely mimics the body position of a road bike as the handlebar position is lower and causes the rider to lean forward more. When spinning from a seated position, exertion is focused on the large muscle groups, namely the quadriceps and gluteal muscles. ${ }^{2}$ Therefore, the commonest complaint of those suffering from overexertion and rhabdomyolysis is thigh pain.

In the literature, a large majority of cases reported were women. ${ }^{2-5}$ This is in contrast to exertional rhabdomyolysis across all other sports that occur more frequently in men. ${ }^{6}$ This may reflect the popularity of spinning among women or temporal trends in class enrolment, rather than any predisposition of females for SIR. Most cases of SIR presented after the first spin class, which is consistent with our study ${ }^{2,5,7}$ and reflect the increased risk of overexertion in spinning for those who are not accustomed to high-intensity workouts.

The average time of presentation to the ED is 3 days after the spin class, which is concerning. Patients may have attributed symptoms of thigh pain to unaccustomed exercise and the effect of a good workout, leading to delayed attention. All but 1 patient presented to the ED only after the onset of brown or dark urine, which occurred 2-3 days after the spinning session. One patient even presented to our ED after receiving treatment for a urinary tract infection. We extrapolated that she was treated presumptively for this due to the presence of haematuria, which also occurs in rhabdomyolysis due to myoglobinuria. These delayed presentations reflect a lack of awareness among beginners on the possibility of rhabdomyolysis following spinning. Many cases may also have been missed. 


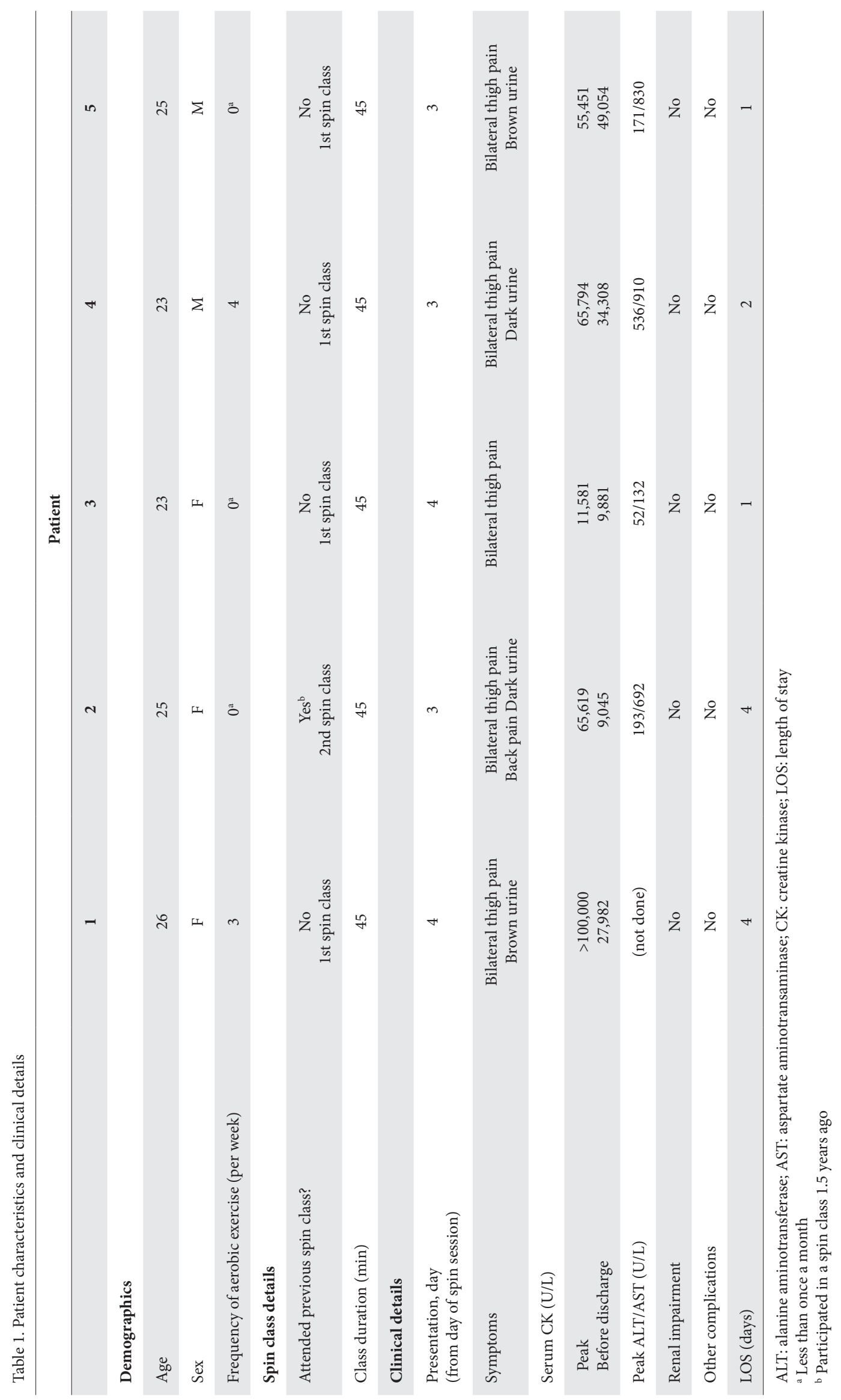


In our literature review, most patients with SIR do well following aggressive hydration with intravenous fluids. However, there have been rare cases of renal failure requiring haemodialysis, and compartment syndrome of the thighs requiring fasciotomy. ${ }^{1,7,8}$

Compared to outdoor cycling, external factors that may decrease the intensity of exercise such as the need to turn, avoid obstacles or stop for traffic are removed in spin cycling. Spin cycling can also be done in a comfortable air-conditioned environment that is free of obstacles, where participants are kept engaged with fast tempo music. Videos may also be projected during sessions, heightening engagement or even distraction from exhaustion. Dimmed lights may also make participants feel less self-conscious and push them to exercise harder. They are also encouraged to "ride with the pack" and teamwork is often emphasised. It is possible that a combination of these conditions will push a newcomer to go beyond his or her physical limit.

Preventive measures. We suggest the grading of spin classes and advocate lower levels of intensity for initial sessions. More attention should be given to participants who are new to the sport, as well as those with sedentary lifestyles. Instructors should exercise extra care towards these participants, as they are more likely to push themselves beyond their limit. Participants should be educated on the risk of overexertion and its complications, and be informed of preventive measures such as adequate warm-up, hydration and nutrition. ${ }^{6}$ Finally, spin class should not be advertised as merely casual exercise and a trendy fad, but instead, as a highintensity workout.

This article was written to raise awareness on the increasing number of SIR cases among beginners. SIR can occur after just a brief period of intense spinning in an untrained person. It may be under-reported as severe complications are rare, and participants are usually young and healthy. We advocate setting an advisory to inculcate safe practice especially for beginners, such as through recommendations for graded training, awareness of overexertion among trainers and participants, and pre-/ post-exercise preventive measures.

\section{REFERENCES}

1. Brogan M, Ledesma R, Coffino A, et al. Freebie rhabdomyolysis: A public health concern. Spin class-induced rhabdomyolysis. Am J Med 2017;130:484-7.

2. Kim D, Ko EJ, Cho H, et al. Spinning-induced rhabdomyolysis: Eleven case reports and review of the literature. Electrolyte Blood Press 2015;13:58-61.

3. Young IM, Thomson K. Spinning-induced rhabdomyolysis: a case report. Eur J Emerg Med. 2004;11:358-9.

4. Kim YH, Ham YR, Na KR, et al. Spinning: an arising cause of rhabdomyolysis in young females. Intern Med J 2016;46:1062-8.

5. Elliott A, Burke R, Liggett N. No pain no gain? Two cases of spin class induced rhabdomyolysis. Ulster Med J 2015;84:132-3.

6. Kim J, Lee J, Kim S, et al. Exercise-induced rhabdomyolysis mechanisms and prevention: A literature review. J Sport Health Sci 2016;5:324-33.

7. DeFilippis EM, Kleiman DA, Derman PB, et al. Spinning-induced rhabdomyolysis and the risk of compartment syndrome and acute kidney injury: two cases and a review of the literature. Sports Health 2014;6:333-5.

8. Cutler TS, Defilippis EM, Unterbrink ME, et al. Increasing incidence and unique clinical characteristics of spinning-induced rhabdomyolysis. Clin J Sport Med. 2016;26:429-31.

Rupeng Mong ${ }^{1}$ MRCEM (UK), Shin Ying Thng ${ }^{1} \operatorname{MRCSEd}(A \& E)$, Shu Woan Lee ${ }^{1}$ FRCSEd (A\&E)

${ }^{1}$ Accident and Emergency Department, Changi General Hospital, Singapore

Correspondence: Dr Rupeng Mong, Accident and Emergency Department, Changi General Hospital,

2 Simei Street 3, Singapore 529889.

Email: mong.rupeng@singhealth.com.sg 